\title{
Long-term consumption of high-fat diet impairs motor coordination without affecting the general motor activity
}

\author{
Andrea Stojakovic ${ }^{1}$, Claudio A Mastronardi ${ }^{2,3}$, Julio Licinio $^{4,5}$ and Ma-Li Wong ${ }^{6,7,7 *}$ \\ ${ }^{1}$ Mayo Clinic, Department of Neurology, 200 First St. SW, Rochester, MN 55905, USA \\ ${ }^{2}$ School of Medicine and Health Sciences, Universidad Del Rosario, Bogota, Colombia \\ ${ }^{3}$ Neuroscience (NEUROS) Research Group, Universidad del Rosario, Bogota, Colombia \\ ${ }^{4}$ College of Medicine, State University of New York Upstate Medical University, Syracuse, NY, USA \\ ${ }^{5}$ Department of Psychiatry, State University of New York Upstate Medical University, Syracuse, NY, USA \\ ${ }^{6}$ Mind and Brain Theme, South Australian Health and Medical Research Institute, Adelaide, Australia \\ ${ }^{7}$ Department of Psychiatry, College of Medicine and Public Health, Flinders University, Bedford Park, Australia \\ ${ }^{8}$ Department of Psychiatry, State University of New York Upstate Medical University, Syracuse, NY, USA
}

\begin{abstract}
Consumption of affordable food that is rich in fat and carbohydrates has increased over the last few decades. As such, high caloric intake and weight gain contribute to sedentary lifestyle. The aim of this study was to investigate the long-term effect of food reach in fat on motor function and dopaminergic system. In the present study, six-week old male C57b1/6 mice were fed either high-fat diet (HFD) or regular chow diet (RD) for 15 months, followed by estimation of spontaneous locomotor activity, body composition, assessment of metabolic outcomes, and histological examination of dopaminergic neurodegeneration. Although body weight stayed similar in both diet-fed mice, HFD-fed mice had decreased lean tissue mass and increased fat mass. In particular, isolation of fat depots showed that HFD-fed mice had increased brown, retroperitoneal and subcutaneous fat mass, in comparison to WT mice. Reduced lean tissue did not influence the muscle strength as it was shown by grid test. The HFD intake did not affect the spontaneous locomotor activity. However, HFD-fed mice displayed time-dependent reduction in motor coordinative skills measured by rotarod, and this was further confirmed by pole test, stepping test and hind-limb clasping test towards the end of in vivo studies. Reduction in coordinative abilities of HFD-fed mice was accompanied by impaired glucose tolerance test, elevated blood insulin and leptin levels. Histological data showed that HFD consumption did not induce loss of tyrosine hydroxylase (TH)-positive dopamine neurons, nor increased number of activated CD68-positive microglia cells in substantia nigra (SN). Taken together, these data suggest that adiposity and insulin resistance induced with HFD consumption led to reduced motor coordination.
\end{abstract}

\begin{abstract}
Abbreviations: ABC: avidin-biotin complex; AEEC: Australian National University Animal Experimentation Ethics Committee; CNS: central nervous system; DA: dopamine; DAB: 3,3`'-diaminobenzidine; GTT: intraperitoneal glucose tolerance test; i.p.: intraperitoneal; IL-1: interleukin-1; IL-1R1: IL-1 receptor 1; IL-1 ra $^{-/}$: IL-1 receptor antagonist knockout; IL-1ß: IL-1 beta; IL-1 $\alpha$ : IL-1 alpha; LPS: lipopolysaccharide; PBS: phosphate-buffered saline; PD: Parkinson's disease; rpm: revolutions per minute; SEM: standard error of the mean; SN: substantia nigra; SNpc: substantia nigra pars compacta; SNpr: substantia nigra pars reticulata; TH: tyrosine hydroxylase; VTA: ventral tegmental area; WT: wild-type.
\end{abstract}

\section{Introduction}

Obesity and ageing are two factors identified in human population to be important contributors to development of neurodegenerative disorders. Feeding the animals with high calorie diet results in significant weight gain with concomitant occurrence of various metabolic problems such as type-2 diabetes, glucose intolerance, insulin resistance [1-3], and dyslipidemia [4]. Diet-induced obesity produces metabolic inflammation that can further upregulate inflammatory cascade in the central nervous system (CNS) via activation of microglia cells [5-7]. During the aging process, primed microglia may release more proinflammatory cytokines and are suggested to become overresponsive [8,9]. Obesity and HFD-induced insulin resistance contribute to impairment of nigrostriatal function and also favor an increase of the susceptibility to loss of dopamine neurons [10-13]. In particular, insulin resistance has been negatively correlated with dopamine release and its clearance from synaptic cleft in HFD-fed mice [11]. It was demonstrated that patients with diabetes and hyperglycemia display poor motor coordination and reduction in general motor activity [14-16]. Along with development of diabetes and insulin resistance in state of obesity, accumulating adipose tissue synthesizes and releases high amount of hormone leptin into the circulation. Studies conducted in rats showed that there might be direct correlation between high levels of leptin and down-regulation of TH enzyme expression [17]. Objective of this study was to assess cumulative effect of aging and long-term HFD consumption in mice on locomotor and coordinative abilities in conjunction with potential loss of dopamine neurons. Here we report that prolonged HFD feeding in mice led to decline in their

${ }^{*}$ Correspondence to: Ma-Li Wong, Professor, 3302 Psychiatry and Behavioral Sciences Building, 713 Harrison Street, Syracuse, NY 13210, USA, Tel. 315 4645540, E-mail: WongMa@upstate.edu

Key words: motor coordination, high-fat, dopaminergic neuron, microglia, diabetes

Received: October 19, 2018; Accepted: October 24, 2018; Published: October 26, 2018 
motor coordination, without propagating activation of microglia and promoting degeneration of dopamine neurons.

\section{Materials and methods}

\section{Animals}

Six-week old male C57bl/6 mice (10 per experimental group) were housed in groups of up to five individuals per cage and maintained under standard living conditions $\left(22+/-2^{\circ} \mathrm{C}, 12 / 12\right.$ hour light / dark cycle, food and water ad libitum). These conditions remained consistent throughout the experiment unless indicated otherwise. The mice were fed either HFD or RD for 15 months. All animal experiments were performed according to the rules and regulations of the "Australian code of practice for the care and use of animals for scientific purposes". All experimental protocols were approved by the Australian National University Animal Experimentation Ethics Committee (AEEC).

\section{Feeding}

Six-week-old male mice were allowed free access to either HFD (18.6\% of fat content and $44.3 \%$ carbohydrates supplied from Specialty Feed (Australia, cat number: SF10-020); this diet is equivalent to Harlan Teklad diet, cat number: TD.95217) or RD (4.8\% of fat content, and $59.4 \%$ carbohydrates supplied from Specialty Feed (Australia, cat number: SF10-020).

\section{Behavioral tests}

Behavioral tests were performed from 8 weeks of age (twice a month) and onwards up to 12 months of age.

Open-field test: Locomotion, coordination and balance skills were evaluated when mice were young (42-45 day old), and 9 months later (i.e. when they became over 10 month old). Locomotion was determined by placing each individual mouse in an open-field arena $(48 \mathrm{~cm} \mathrm{x} 48 \mathrm{~cm})$ and recording its activity for 32 minutes with a video camera placed from the top of the arena. The software "Viewer 3" (Biobserve Gmbh, St. Augustin, Germany) was used for data collection and processing, which provided measures of the total distance travelled.

Rotarod test: Coordination and balance skills were ascertained by employing a rotarod apparatus (Panlab, Harvard apparatus, Barcelona, Spain). Prior to starting data collection, mice were trained for 4-6 days and were given 3 trials per day in order to achieve maximal performance in the rotarod test [18-20]. Mice were given 3 trials with 2-minute breaks between trials. Upon placing the mice on the rotating drum, the initial speed was 4 revolutions per minute (rpm), and it was accelerated to $40 \mathrm{rpm}$ within 2 minutes. The latency to fall from the rotating drum was measured in seconds. Mice that showed better balance and coordination skills were able to remain for a longer period of time on the rotating drum. The final result was calculated as the mean of the latency to fall from the rotarod apparatus during the 3 trials. In the infrequently observed event in which the mouse had not fallen from the rotating drum after 2 minutes, it was removed from the apparatus and returned to its home cage [21]. During the training period all mice were fed a $\mathrm{RD}$. All of the mice were included since they were successfully trained for this behavioral paradigm, and they looked healthy throughout the entire duration of the study as per routine inspections of their fur, body weight, response to handling, and home cage social behavior. The mice were frequently monitored by the researchers and technical staff of the Australian National University animal facility.

Grid test (the four limb hang test): The grid test is used to evaluate the strength of mouse limbs $[22,23]$. This test was used as a control test to determine if the observed differences in motor coordination displayed by different mice in the rotarod test could be due to reduced muscle strength. The test aims at determining the ability of the animal to grasp the wire mesh with both forelimbs and hind limbs and to remain clinging in the inverted positions for a period of time. The latency to fall was recorded in seconds and analyzed. Mice were given three trials, separated by intertribal intervals of 5 minutes. The height of the apparatus is between $20-50 \mathrm{~cm}$ to prevent that the animal climbs down easily.

Pole test: This test represents one of the possible methods used to assess the agility of the animals. It is mostly used as a measurement of bradykinesia (slowness of movement) [24]. The test requires the usage of pole, 50 to $55 \mathrm{~cm}$ in height and 8 to $10 \mathrm{~mm}$ in diameter with rough surface that stands vertically in a home cage. The test was conducted by placing an animal close to the top of the pole and leaving the animal's head up. The period of time that animal spent in turning around (the first measurement) and climbing down (the second measurement) the pole was recorded. Therefore, both parameters (time to turn around and climb down) were used for assessment of bradykinesia in mice.

Stepping test: The stepping test is another reliable method to assess forelimb akinesia [25]. It is performed on a table and the animal is placed on one edge of it. Afterwards, hind legs are lifted by pulling animal's tail upwards and in that position the animal is pulled back, toward the other edge of the table in length of 1 meter. The number of animal's adjusting steps from both forepaws are counted [25].

Hind limb clasping test: This test is used for an assessment of dyskinesia (diminished voluntary movements) in mice [26]. Mice are suspended in the air by the tail for a period of ten seconds. The position of hind limbs is observed and scored. Based on the position of the limbs mice are scored as:

0 - hind limbs are splayed outwards,

1- one hind limb is retracted towards abdomen,

2- both hind limbs are partially retracted towards abdomen and [27].

3- hind limbs are completely retracted and touching the abdomen

\section{Metabolic tests}

Food intake and body weight measurement: Food intake was monitored once a week, for seven months. At the end of every seven days, the leftover food from the cage was weighted and recorded. Food intake was expressed as an average of food weight (in kcal) per animal and normalized by animal body weight (BW). The mouse's body weight was measured weekly over a period of thirteen months. Mice were weighted and their body weight was recorded. Final results were expressed as a mean value of total number of animals/per group. Towards the end of in vivo experiments, body composition (percentage of fat mass and weight of lean mass) was determined by DEXA scan.

DEXA scan: Dual-emission X-ray absorptiometry (DEXA) scan is used primarily to evaluate bone mineral density (BMD), body composition and fat content [28]. Prior to testing, animals are deeply anesthetized with 2-3\% isofluorane-oxygen gas for an entire period of test (5-10 minutes) and then placed in a PIXImus apparatus (PIXImus Mouse Densitometer). The whole body of each mouse was scanned and analyzed with PIXImus software (LUNAR PIXImus 2). The mouse head was excluded from the scan by using the ROI (region of interest) that was placed manually surrounding the whole body. The data that 
were used for further analysis were: weight and percentage of fat mass, weight of lean tissue.

Intraperitoneal glucose tolerance test (IPGTT): The IPGTT test is used to assess how fast the glucose is cleared out from the blood upon glucose (i.p.) injection. Prior to testing, mice were transferred to clean cages and fasted for a period of 6 hours, but they were provided with water ad libitum. Mice were weighed and their baseline blood glucose levels were checked by collecting one small drop of blood from animal's tail vein at 0 minute (baseline). Afterwards, mice were injected with D-glucose (2 g/kg, i.p.) solution [29], which was previously dissolved in phosphate buffered saline (PBS). The glucose levels were measured by a glucometer (Accu Chek(R), Roche, Australia) at 30, 60, 90 and 120 minutes after the injection. At the end of 14 months of mice age, mice were subjected to intraperitoneal glucose tolerance test in order to assess their glucose tolerance according to their treatment. Two months later, mice were euthanized and their blood collected and plasma levels of leptin and insulin were assessed by ELISA method.

The enzyme-linked immunosorbent assay (ELISA): Blood was collected transcardialy in ethylenediaminetetraacetic acid (EDTA)coated tubes. Blood samples were centrifuged at $2000 \mathrm{~g}$ for 15 minutes at $4{ }^{\circ} \mathrm{C}$ and plasma was separated from blood cloth and stored at $-20^{\circ} \mathrm{C}$ until used. The samples for insulin measurement were diluted in ratio of $1 / 8$ for HFD-fed mice, while samples of RD were not diluted. Plasma insulin was measured with ALPCO ELISA kit (Mouse Ultrasensitive Insulin ELISA, catalog number: 80-INSMSU-E01, E10). Leptin levels were measured with mouse Leptin ELISA kit (DuoSet R\&D Systems, catalog number: DY498). Samples for leptin measurements were diluted in ratio $1 / 3$ for $\mathrm{RD}$-fed mice and $1 / 8$ for HFD-fed mice.

Dissection of adipose tissues: Brown adipose tissue was isolated from the dorsal aspect of the thorax between the scapulae and collected in pre-weight ependorf tubes and weight was measured after the freezing in dry ice. The epididymal, retroperitoneal, visceral and brown fat pads were manually isolated and weighted.

\section{Immunohistochemistry of dopamine neurons and microglia cells}

Tissue preparation: Fifteen months after the initiation of the experiments, mice were anesthetized with an intraperitoneal injection of ketamine/xylazine $(100 / 10 \mathrm{mg}$ per $\mathrm{kg}$, adjusted to a volume of $0.1 \mathrm{ml} / 10 \mathrm{~g}$ of body weight), and transcardially perfused with a solution of cold phosphate-buffered saline (PBS) and heparine $(5 \mathrm{U} / \mathrm{ml})$ within 3 minutes. The mouse brain was removed and frozen in chilled isopentanol and stored at $-80^{\circ} \mathrm{C}$ until use. Brains were sliced using a cryostat (set at $-12^{\circ} \mathrm{C}$ ) in $15 \mu \mathrm{m}$ coronal sections throughout the entire SN.

Immunohistochemistry: We followed previously reported protocols for tyrosine hydroxylase (TH) and microglia staining with minor modifications [30,31]. Brain sections were collected on gelatin-coated slides, air-dried and post-fixed in $4 \%$ paraformaldehyde (pH 7.4 in PBS) for 8-10 minutes. After a PBS wash (3 times within 5 minutes), sections were incubated for 20 minutes in $3 \%$ peroxide mixed in methanol in order to inactivate the endogenous peroxidase. Sections were incubated with $5 \%$ normal goat serum and $0.015 \%$ Triton X-100 in PBS. After 30 minutes of incubation, the sections were incubated with rabbit anti-TH antibody (1:1000 dilution; Life Technology, cat\# P21962) [30], or rat anti-mouse CD68 monoclonal antibody (1:700; AbD Serotec, cat\# MCA341GA) in $0.015 \%$ Triton X-100 for 24 hours at $4^{\circ} \mathrm{C}[31]$. On the following day, the slides were washed in PBS and incubated with biotinylated goat anti-rabbit (1:200; Vector Laboratories, cat\# PK-6101) or goat anti-rat secondary antibody (1:500; Vector Laboratories, cat\# BA-9400) for 1 hour. After a PBS wash, slides were reacted with avidin-biotin complex (ABC; Vector Laboratories, cat\# PK-6101) for 30 minutes. In the final step, slides were washed with PBS and incubated with 3,3' '-diaminobenzidine (DAB; Sigma, cat\# D3939) and $30 \%$ peroxide until color development (5-7 minutes). Slides were counterstained with hematoxylin (30 seconds), dehydrated in alcohol gradient $(80 \%, 90 \%, 100 \%, 100 \%)$, immersed in xylene, mounted with DPX slide mounting medium (Sigma, cat\# 05622), and coverslipped. All the incubations were done at room temperature, unless mentioned otherwise.

Quantification of dopamine neurons and microglia: In order to assess the loss of dopamine neurons and the number of activated of microglia cells, two adjacent series of 8 consecutive slides $(15 \mu \mathrm{m}$ of thickness) were collected to sample the region of SN (rostral to caudal: -2.65 to $-3.61 \mathrm{~mm}$ posterior to bregma) [21]. Thus, the two series of eight evenly spaced slides were obtained every $90 \mu \mathrm{m}$, and were used for counting dopamine neurons or activated microglia cells. The borders of SN were defined in order to exclude the ventral tegmental area (VTA) from counting [32]. The area defined for counting covered the entire area from the rostral part of $\mathrm{SNpc}$ to the caudal end of $\mathrm{SN}$ pars reticulata (SNpr), excluding those TH neurons that interspersed with oculomotor nerve rootlet. Images were obtained with an IX2-UCB Olympus digital camera (Olympus, Tokyo, Japan), and analyzed manually using the ImageJ software (http://imagej.nih.gov from NIH). Positive CD68 microglia cells in SN were counted manually on a Nikon Eclipse 50i microscope (Nikon, Tokyo, Japan). The picture magnifications were 20x and 40x for microglia and 20x for $\mathrm{TH}$.

\section{Statistical analysis}

Data were analyzed with Graph-Pad software version 5 (GraphPad Software, La Jolla California USA) and expressed as Mean \pm standard error of the mean (S.E.M). Time-dependent differences in behavioral and metabolic outcomes were analyzed by unpaired Student's T-test, and two-way ANOVA. Values of $P<0.05$ were considered significant.

\section{Results}

\section{Long-term effect of high-fat diet consumption on locomotor activity}

HFD-fed mice did not show significant decline in general motor activity in open-field test in comparison to RD-fed group (Figure 1a). Baseline performance of RD and HFD-fed mice in the rotarod test was not different. However, motor coordination with HFD consumption progressively declined over the course of nine months (Figure 1b). Overall, diet comparison within groups showed that the extent of coordination impairment observed in HFD-fed mice was markedly greater in comparison to RD-fed mice, whereas general motor ability was not affected by HFD consumption.

The pole test is a useful method for assessment of bradykinesia, which describes slowness of a performed movement, and it has been shown to correlate with the levels of dopamine in striatum [33]. Comparison between two different diet-fed groups indicated that HFD-fed mice tended to spend more time to turn around on the pole accompanied with significant delay to climb down (Figures $2 \mathrm{a}$ and $2 \mathrm{~b}$ ). As it was previously described, the stepping test is a reliable test used to determine forelimb akinesia, a movement disorder that has been related with nigrostriatal loss of dopamine [34]. HFD-fed mice showed 
significant reduction in their ability to adjust forelimbs steps $[41 \%$ $(P<0.001)]$ (Figure 2c). Evaluation of dyskinesia (diminished voluntary and prevalence of involuntary movements) may be conducted by performing a hind limb clasping test. The hind limb clasping test is performed by suspending mice in the air by the tail and the position of hind limbs are observed. Based on the position of the limbs HFDfed mice showed signs of dyskinesia in comparison to RD mice (Figure 2d). One of the experiments performed after the open-field and rotarod test was the grid test, which gives reliable indications on the general condition of limb muscle strength [35]. The test was performed in order to exclude the possible effects of muscle strength disability that might occur in mice fed a HFD, which would have in turn affected their rotarod performance. The overall time that animals spent hanging on the horizontally positioned grid indicated that muscle strength was even
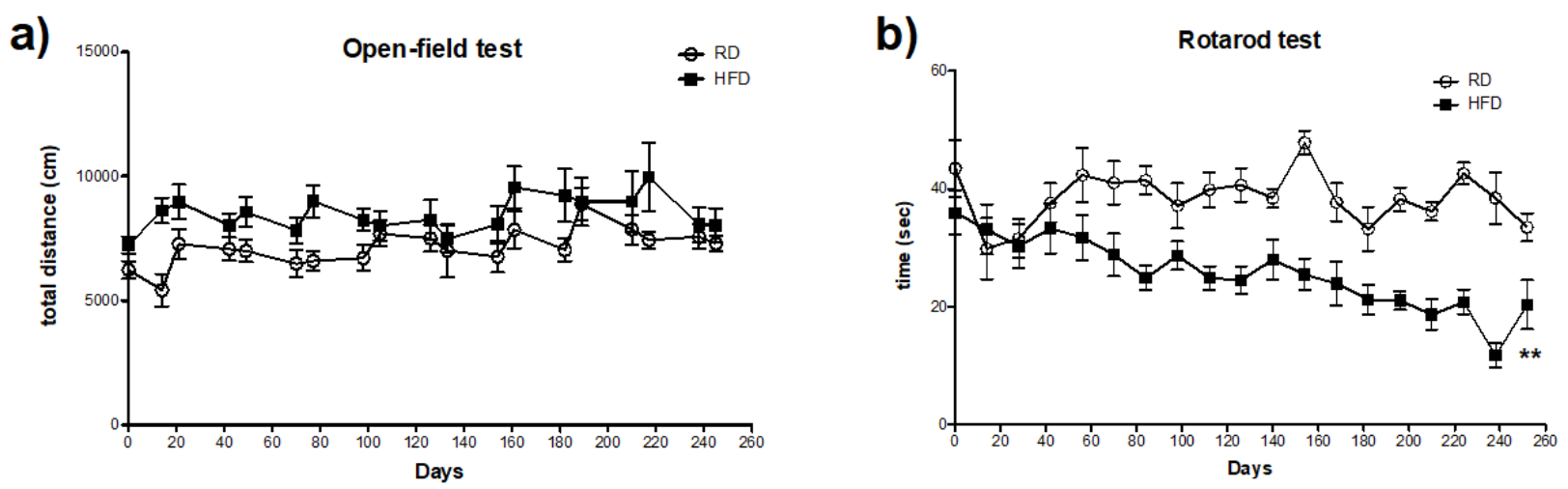

Figure 1. Assessment of locomotor activity. (a) Total distance travelled during open-field test. Comparison of the total distance travelled by RD ( $\mathrm{n}=10$ ) and HFD ( $\mathrm{n}=12$ ) mice within period of 9 months. Statistical comparison between two groups was performed with two-way ANOVA followed. (b) Rotarod performance. Comparison of the average latency (measured in seconds) to fall from the rotating drum displayed by $\operatorname{RD}(\mathrm{n}=10)$ and $\operatorname{HFD}(\mathrm{n}=12)$ during 9 months test period. Statistical comparison between two was performed with two-way ANOVA. $* * P<0.01$ vs. $\mathrm{RD}$

a)

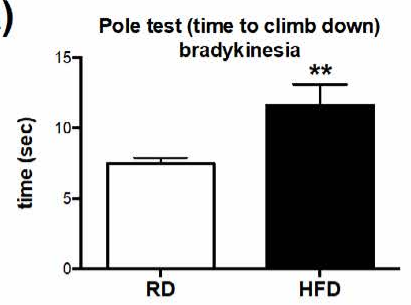

c)

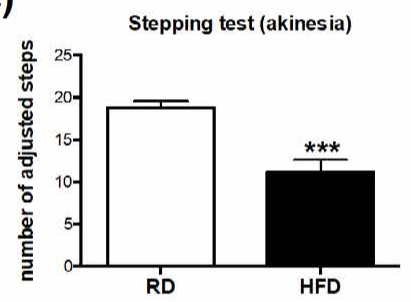

e)

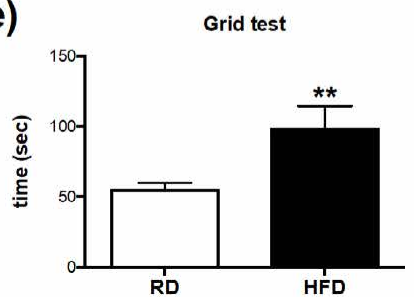

b)

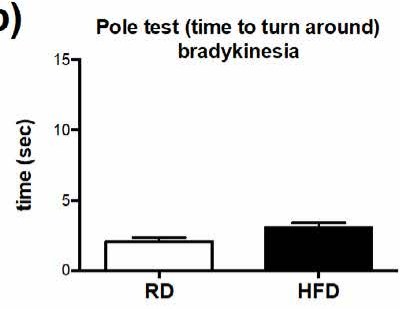

d)

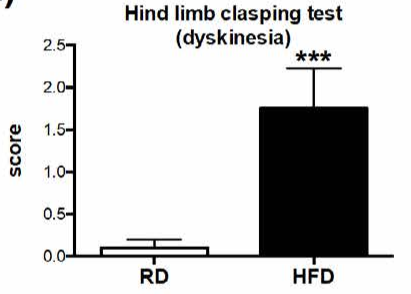

Figure 2. Assessment of coordinative abilities: Pole test: (a) Latency for mice fed either RD ( $\mathrm{n}=10)$, or HFD ( $\mathrm{n}=5$ ) to turn around and (b) climb down the pole was recorded in seconds. (c) Mice performance in the stepping test: number of adjusted step in mice fed either RD $(n=10)$, or HFD ( $\mathrm{n}=4)$ was measured in length of $1 \mathrm{~m}$ and recorded as average count of adjusted forelimb steps over three given trials for each mouse. (d) Hind limb clasping test: mice fed either RD ( $\mathrm{n}=10)$ ), or HFD ( $\mathrm{n}=4$ ) were suspended in the air for 10 seconds by the tail and their performance to clasp hind limbs was scored. (e) Mice performance in the grid test: Limb muscle strength was measured as the latency for mice fed with either RD ( $\mathrm{n}=10$ ), or HFD ( $\mathrm{n}=4$ ) to fall down from horizontally positioned metal grid of the apparatus and recorded in seconds. Error bars represent standard errors All data were analyzed by unpaired T-test. $* * P<0.01$, $* * * P<0.001$ vs. RD 
better in HFD-fed mice in comparison to RD (Figure 2e), which suggest that long-term consumption of HFD did not impair muscle strength in the mice. Collectively, these data suggest that long-term exposure to HFD might have impaired dopaminergic neurotransmission.

\section{Long-term effect of high-fat diet on metabolic outcomes}

There were no significant differences in the initial body weight observed between RD and HFD-fed mice nor after the 13 months collection period (Figure 3a). Food consumption was significantly higher in HFD-fed mice in comparison to RD group (Figure 3b). Similarly, normalized data of food intake versus corresponding body weights of RD and HFD mice in Figure 3c showed that HFD-fed mice had an overall increased caloric intake. These results indicate that in the experimental paradigm HFD consumption increased energy intake.

In order to further evaluate the differences in body composition between RD and HFD-fed group, the percentage and weight of body fat mass, weight of lean mass were assessed by DEXA scan. The diet comparison of body fat mass composition and its percentage showed that HFD-fed mice in general had a higher percentage of fat accumulation in comparison to RD group, despite similar body weight (Figures $4 \mathrm{a}, \mathrm{b}$ and $\mathrm{d}$ ). On the other hand, HFD-fed mice had less
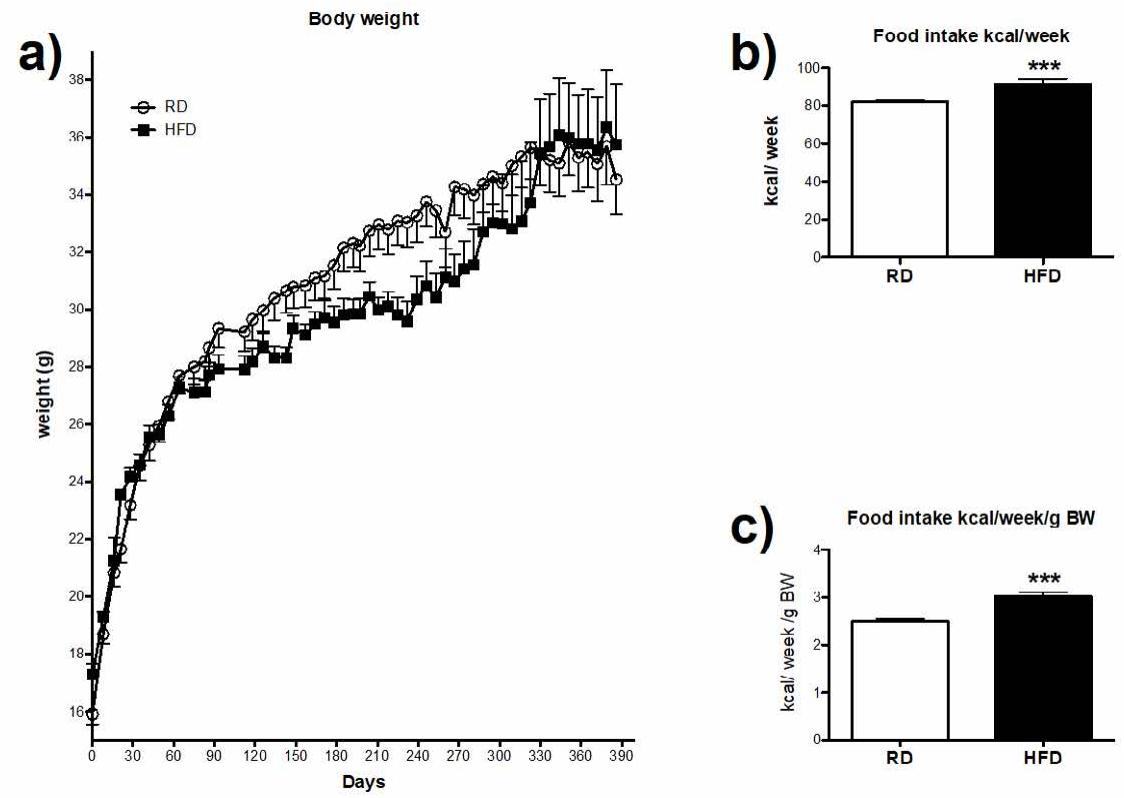

Figure 3. Effects of high-fat diet consumption on body weight and food intake: (a) Body weight data were collected weekly for the period of 13 months. Food intake was measured on a weekly basis. Results were expressed as a mean/per cage, since 3-5 animals were housed in a single cage. (b) Average food intake of RD ( $\mathrm{n}=10$ ) and HFD-fed mice (n=10) collected over a period of seven months. (c) Percentage of food intake normalized by body weight of RD and HFD-fed mice. All data were analyzed by unpaired T-test. Error bars represent standard errors. $* * * P<0.001$ vs. RD
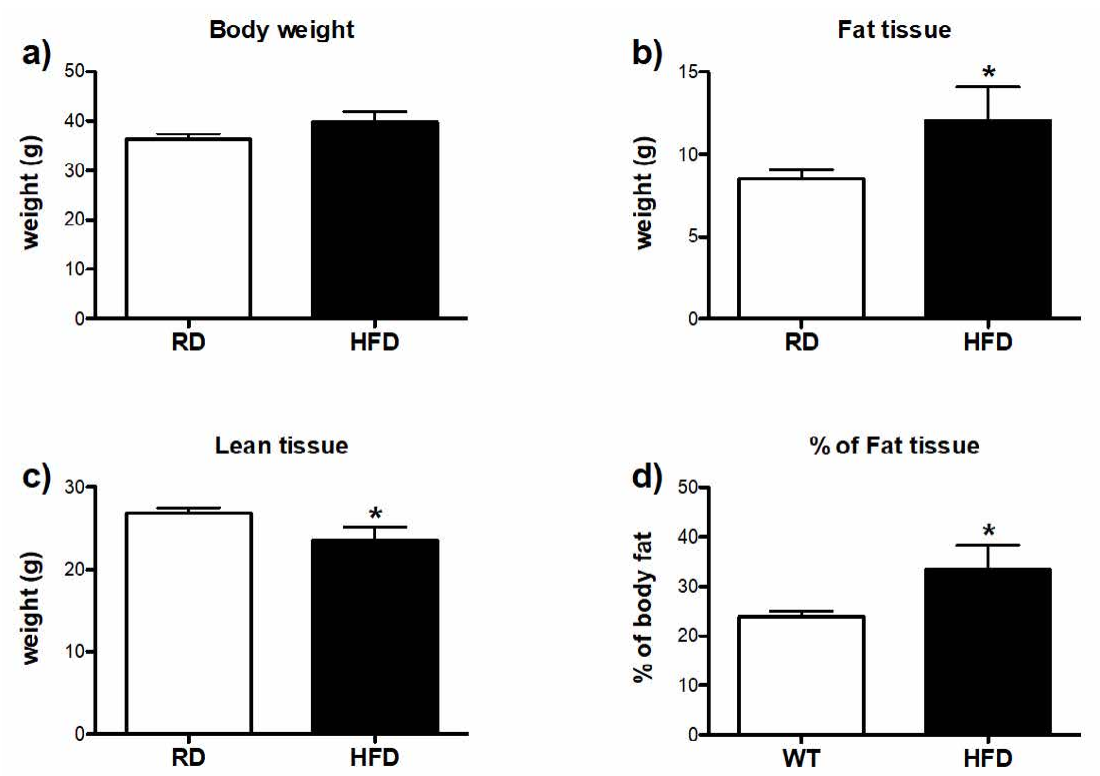

Figure 4. Effects of high-fat diet on fat mass and lean tissue remodeling: (a) Body weight data collected at the time of DEXA scan. (b) Weight and (d) percentage of the fat tissue. (c) Weight of lean tissue. Differences between RD $(n=10)$ and HFD-fed mice $(n=4)$ were analyzed by unpaired T-test. Error bars represent standard errors. $* P<0.05$ vs. RD 
lean tissue (Figure 4c). Detailed examination of individual fat depots revealed that amount of brown (Figure 5a), retroperitoneal (Figure 5b) and subcutaneous (Figure 5c) adipose tissues was higher with longterm HFD consumption, while weight of epididymal fat depot was similar to RD-fed mice (Figure 5d).

In order to assess the additional effects of long-term HFD consumption on metabolic outcomes, IPGTT test, insulin and leptin levels tests were performed. Blood glucose level was determined by
IPGTT test at the end of 15 months of in vivo studies. HFD-fed mice had a higher baseline level of glucose and showed a significant increase in glucose intolerance at 60, 90 and 120 minutes after glucose injection (Figure 6a). Further analysis revealed that HFD-fed mice were glucose intolerant (Figure 6b). Moreover, HFD-fed mice displayed higher levels of insulin than RD mice (Figure 6c), indicating that HFD-fed mice were hyperinsulinemic. Similarly, leptin level was significantly elevated in HFD-fed mice in comparison to RD-fed mice (Figure 6d). Overall, data
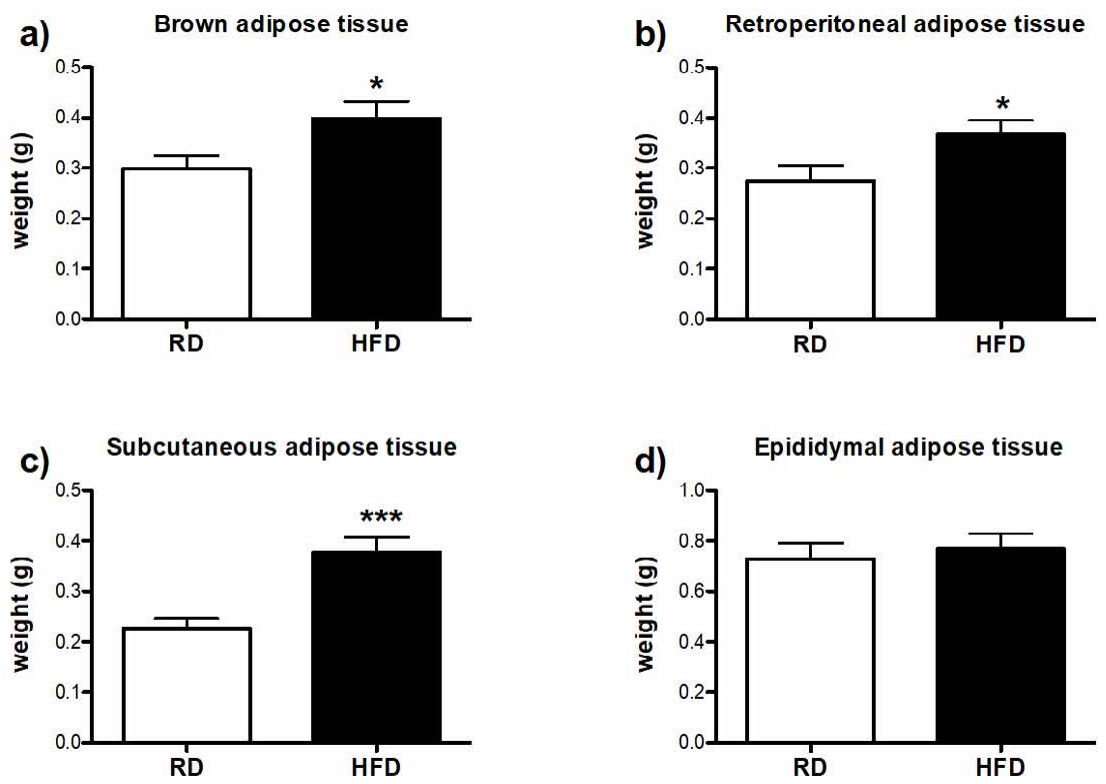

Figure 5. Effect of high-fat consumption on remodeling of individual fat depots. After euthanasia, individual fat depots were collected and weighted. (a) weight of brown fat tissue, (b) retroperitoneal adipose tissue, (c) subcutaneous adipose tissue and (d) epididymal adipose tissue expressed in grams. Differences between RD ( $\mathrm{n}=10)$ and HFD-fed mice ( $=4$ ) were analyzed by unpaired T-test. Error bars represent standard errors. ${ }^{*} P<0.05, * * * P<0.001$ vs. RD

a)

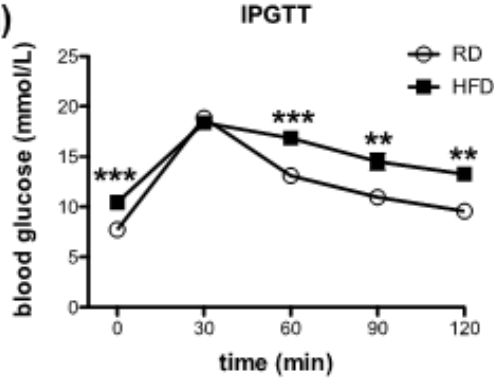

c)

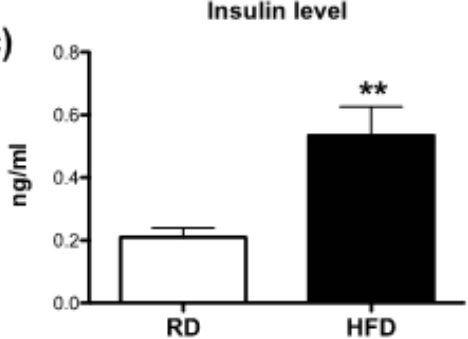

b)

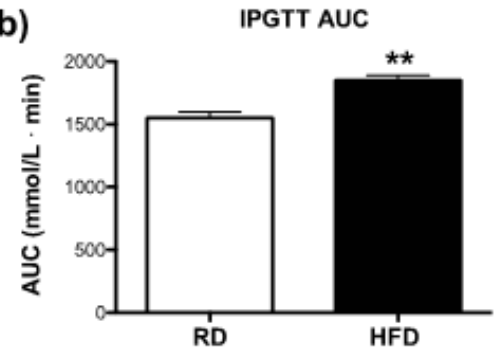

Leptin level

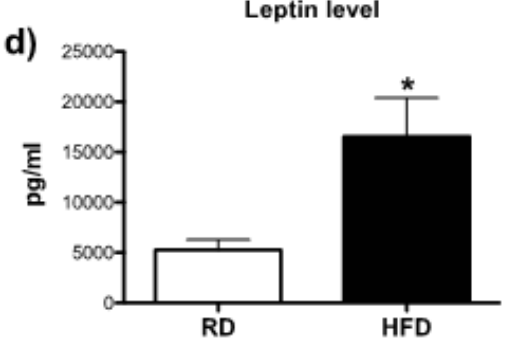

Figure 6. Assessment of metabolic parameters: Effect of high-fat diet on blood glucose level in IPGTT test: Mice were fasted for 6 hours and were injected (i.p.) with 2g/kg of glucose solution [393]. Blood glucose level was measured at 0 min before injection and 30, 60, 90 and 120 min after the injection. (a) Blood glucose levels of RD ( $\mathrm{n}=10$ ) and HFD-fed mice ( $\mathrm{n}=4$ ) were analyzed by one-way ANOVA (Tukey`s multiple comparison test). (b) Glucose tolerance in between RD and HFD-fed mice was analyzed by AUC and differences were compared by unpaired T-test. Level of insulin and leptin in the blood plasma was measured by ELISA kit. Differences in (c) insulin and (d) levels of RD and HFD-fed mice were analyzed by unpaired T-test. Error bars represent standard errors. $* P<0.05, * * P<0.001, * * * P<0.0001$ vs. RD 
presented here are suggesting that HFD-fed mice became metabolically obese despite having similar body weight to RD-fed mice.

\section{Immunohistological examination of high-fat diet effect on degeneration of dopaminergic neurons}

In order to assess the effect of HFD consumption on neuronal degeneration of dopamine neurons, the total number of TH-positive neurons was quantified from eight evenly spaced frozen sections of the brain that encompassed the entire region of the SNpc. Representative pictures displaying positive $\mathrm{TH}$-staining of dopamine neurons in SNpc of RD and HFD-fed mice are shown in Figure 7 (left panels). As presented in the Figure 8a, there was no evident reduction of dopamine neurons in HFD-fed mice in comparison to RD-fed mice.

Further on, activated microglia/macrophages were stained with the CD68 marker and counted in the area of the SNpc and SNpr. A positive CD68-staining of activated microglia cells in the SN of RD and HFD-fed mice is presented in Figure 7 (right panels). As presented in Figure $8 \mathrm{~b}$, quantification of activated microglia yielded no differences between HFD and RD-fed mice, indicating that long-term feeding on HFD did not induce significant upregulation of inflammatory cascade in the $\mathrm{SN}$.

\section{Discussion}

General control over locomotor activity is highly dependent on normal functionality of basal ganglia and sufficient production of dopamine by its neurons. In current study, the long-term feeding on HFD in WT mice did not induce gross motor disabilities. Assessment of voluntary movements in open-field test yielded no difference between HFD and RD-fed mice. Immunohistological quantification of TH-positive neurons showed that long-term HFD consumption did not induce significant loss of dopaminergic neurons and this finding is in the agreement with the open-field results.

Mice coordination has been tested in the rotarod test, and as it was described previously, this test is able to detect an imbalance in the dopamine system [21]. The ability of RD or HFD-fed mice to keep their balance on the rotarod apparatus during baseline assessment was not different between the two groups. During nine months test period, the motor coordination of HFD-fed mice significantly declined in comparison to $\mathrm{RD}$-fed mice. These observations may suggest that this significant decrease in the coordination of HFD-fed mice may have resulted from a decline in the striatal dopamine level. Although the HFD group was fed a fat enriched diet for a several months, these mice did not gain a significant amount of body weight. Thus, decreased rotarod

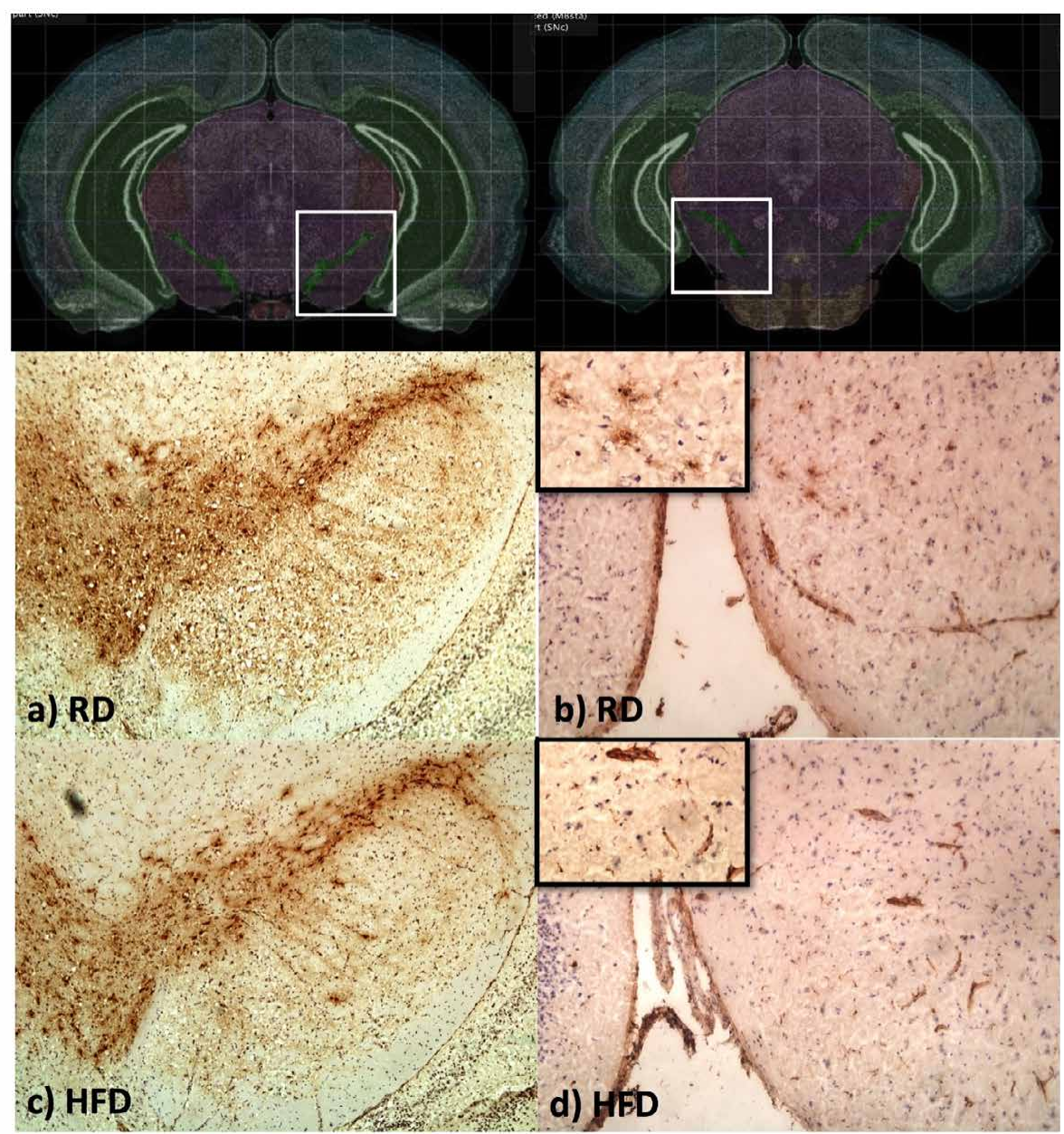

Figure 7. Dopamine neurons and microglia cell count. (a) Mice brains were at 15 months. The total number of TH-positive cells from eight evenly spaced frozen sections that encompassed the entire SN of RD $(n=5)$ and HFD ( $n=7)$ mice were evaluated from the unilateral side of SNpc. (b) Eight evenly spaced frozen sections that encompassed the entire SN of RD ( $\mathrm{n}=5)$ and HFD $(n=6)$ were stained with anti-CD68, a marker for activated microglia. Total count of CD68-positive cells was counted unilaterally in the region of SN. Data were analyzed by student T-test. Error bars represent standard errors 
performance in HFD mice was not due to increased body weight, which was described as one of the elements that may affect the balance on rotarod apparatus [36]. However, these mice were metabolically obese as it was shown by DEXA scan of body composition, IPGTT and greater fat pad mass. Consumption of a HFD did not impair the muscle strength in mice as it was demonstrated with the grid test. Therefore, coordinative disabilities observed in HFD-fed mice could not be attributed to the possible impairment of neuromuscular function. Along with the coordinative impairment demonstrated with rotarod test, further motor assessment showed that HFD-fed mice displayed signs of akinesia, bradykinesia and dyskinesia.

The pole test, as described previously, is used in an assessment of bradykinesia, which is also one of the symptoms related with nigrostriatal dysfunction [33]. Animals that show signs of bradykinesia need more time to turn around and climb down the pole. Results showed that it took significantly more time for HFD-fed mice to climb down the pole. The stepping test was used to assess akinesia, symptom that is characterized by the impairment to initiate steps and it was related with the loss of positive TH-neurons [37]. HFD-fed mice performed significantly worse than RD-fed mice. As described previously, akinesia has been pharmacologicaly induced in mice by inhibition of D2 receptors [38]. Since HFD feeding and obesity in mice was associated with decreased density of D2 receptors [39], lower availability of D2 receptors in HFD-fed mice could have induced symptoms of akinesia. The hind limb clasping test has been used for the evaluation of dyskinesia (presence of involuntary movement and diminished voluntary movements). The comparison between the two groups indicated that HFD-fed mice showed significant neurological/ motor impairment. Clasping behavior has been observed in DAT -/mice [40], implying that the motor impairments observed in HFD-fed mice might be related to a reduced level of dopamine transporters. Overall, coordinative impairments observed in HFD-fed mice might be attributed to decreased level of dopamine in striatum and/or impaired dopaminergic neurotransmission.

In the current study, several metabolic outcomes were assessed in order to understand the possible impact of HFD-induced metabolic disorders on function of dopamine system. Therefore, body weight, glucose tolerance, insulin and leptin levels were measured as contributing factors. Average food consumption was increased in HFD-fed mice and also remained higher relative to body weight. It has been already described that chronic HFD consumption alters the homeostatic regulation of energy balance and leads to hyperphagia $[41,42]$. Moreover, consumption of a HFD positively correlates with accumulation of adipose tissue [43]. Despite increased food intake, HFD-fed mice had similar body weight to RD-fed mice. We hypothesize that frequent handling, measurements and behavioral assessments could have resulted in reduced weight gain in HFD-fed mice. Nevertheless, HFD-fed mice had more accumulated fat tissue, which was expected from the higher food intake. HFD-fed mice showed to be glucose intolerant and had higher plasma levels of insulin and leptin. Obesity can trigger chronic inflammatory and metabolic imbalances affecting dopaminergic neurotransmission. For example, it has been shown that dopamine content was reduced in the nigrostriatal system of diabetic rats [44]. In human studies, diabetes has been shown to augment motor impairment in Parkinson's disease patients [45]. Correlation between type-2 diabetes and Parkinson`s disease was reported in more than 50\% of cases [46]. Insulin injection into mice brains increases the activity of DAT in SN [47], but chronic hyperinsulinemia was shown to reduce the expression of insulin receptors in the blood brain barrier (BBB), which leads to central insulin resistance [48]. Moreover, development of insulin resistance in HFD-fed rats reduced dopamine release and its reuptake in the synaptic vesicles [11].

In regards to leptin, leptin resistance may not influence the basal level of dopamine, but it can reduce the total capacity of dopamine cells to secret dopamine [49]. In fact, it has been shown that leptin regulates the expression of dopamine transporters [50]. Moreover, continued feeding on a HFD induces central leptin resistance accompanied with weight gain [51].

Aging is characterized by increased basal level of proinflammatory cytokines such as IL- $1 \beta$, IL- 6 and TNF- $\alpha$ in the periphery and the brain $[8,52]$. Age-related priming of microglial cells is suggested to facilitate an environment that could be described as a low-grade chronic neuroinflammation [53]. In a similar manner, HFD consumption and obesity are also considered to facilitate a low-grade chronic inflammatory state [54] that could potentiate the up-regulation of peripherally-induced inflammation. A HFD feeding for two months exacerbated the decrease of dopamine in the striatum in the MPTPinduced model of dopaminergic neurodegeneration [13]. Furthermore, it was demonstrated that mice fed a HFD for 22 weeks had an increased level of proinflammatory cytokines in the brain [55]. Even though there was no detectable difference in the number of CD68-positive microglial cells (measured as an index of microglia activation), it has been additionally observed that isolated microglial cells from HFDfed mice secreted more TNF- $\alpha$ in comparison to RD-fed mice [55]. Collectively, these studies suggest that the inflammatory profile in the brain may be up-regulated with HFD-induced obesity and possibly exacerbated the loss of dopamine neurons [13], which does not have to be necessarily correlated with the number of activated microglia [55]. In current study HFD diet feeding did not result in increased number of activated microglial cells, nor significant loss of dopaminergic neurons. Therefore, time-related decrease in coordinative ability of HFDfed mice could be attributed to potentially impaired dopaminergic neurotransmission, due to perturbations in metabolic outcomes, such as development of leptin and insulin resistance.

\section{Acknowledgments}

This study was financed by institutional funds from the John Curtin School of Medical Research, The Australian National University.

\section{References}

1. Lavau M, Fried SK, Susini C, Freychet P (1979) Mechanism of insulin resistance in adipocytes of rats fed a high-fat diet. J Lipid Res 20: 8-16. [Crossref]

2. Klöting N, Fasshauer M, Dietrich A, Kovacs P, Schön MR, et al. (2010) Insulinsensitive obesity. Am J Physiol Endocrinol Metab 299: E506-515. [Crossref]

3. Li P (2015) LTB4 promotes insulin resistance in obese mice by acting on macrophages, hepatocytes and myocytes. Nat Med 21: 239-247.

4. Grundy SM (2004) Obesity, metabolic syndrome, and cardiovascular disease. J Clin Endocrinol Metab 89: 2595-2600. [Crossref]

5. Thaler JP (2013) Hypothalamic inflammation: Marker or mechanism of obesity pathogenesis? Diabetes 62: 2629-2634.

6. Valdearcos M, Robblee MM, Benjamin DI, Nomura DK, Xu AW, et al. (2014) Microglia dictate the impact of saturated fat consumption on hypothalamic inflammation and neuronal function. Cell Rep 9: 2124-2138. [Crossref]

7. Erion (2014) Obesity elicits interleukin 1-mediated deficits in hippocampal synaptic plasticity. J Neurosci 34: 2618-2631.

8. Godbout JP (2005) Exaggerated neuroinflammation and sickness behavior in aged mice following activation of the peripheral innate immune system. Faseb j 19: 1329-1331.

9. Dilger RN, Johnson RW (2008) Aging, microglial cell priming, and the discordan central inflammatory response to signals from the peripheral immune system. J Leukoc Biol 84: 932-939. 
10. Bruce-Keller AJ, Keller JN, Morrison CD (2009) Obesity and vulnerability of the CNS. Biochim Biophys Acta 1792: 395-400. [Crossref]

11. Morris JK (2011) Insulin resistance impairs nigrostriatal dopamine function. Exp Neurol 231: 171-180.

12. Morris JK, Bomhoff GL, Stanford JA, Geiger PC (2010) Neurodegeneration in an animal model of Parkinson's disease is exacerbated by a high-fat diet. Am J Physio Regul Integr Comp Physiol 299: R1082-1090. [Crossref]

13. Bousquet M, St-Amour I, Vandal M, Julien P, Cicchetti F, et al. (2012) High-fat diet exacerbates MPTP-induced dopaminergic degeneration in mice. Neurobiol Dis 45: 529-538. [Crossref]

14. Daneman D (2001) Diabetes-related mortality. A pediatrician's view. Diabetes Care 24: 801-802. [Crossref]

15. Cox DJ (2005) Relationships between hyperglycemia and cognitive performance among adults with type 1 and type 2 diabetes. Diabetes Care 28: 71-77.

16. Brach JS (2008) Diabetes mellitus and gait dysfunction: Possible explanatory factors. Phys Ther 88: 1365-1374.

17. Li Y (2009) High-fat diet decreases tyrosine hydroxylase mRNA expression irrespective of obesity susceptibility in mice. Brain Res 1268: 181-189.

18. Zhu BG, Sun Y, Sun ZQ, Yang G, Zhou CH, et al. (2012) Optimal dosages of fluoxetine in the treatment of hypoxic brain injury induced by 3-nitropropionic acid: implications for the adjunctive treatment of patients after acute ischemic stroke. CNS Neurosci Ther 18: 530-535. [Crossref]

19. Yamada MH, Nishikawa K, Kubo K, Yanagawa Y, Saito S (2012) Impaired glycinergic synaptic transmission and enhanced inflammatory pain in mice with reduced expression of vesicular GABA transporter (VGAT). Mol Pharmacol 81: 610-619. [Crossref]

20. Kreutzfeldt M1, Bergthaler A, Fernandez M, Brack W, Steinbach K, et al. (2013) Neuroprotective intervention by interferon- $\hat{\mathrm{I}}^{3}$ blockade prevents CD8+ T cell-mediated dendrite and synapse loss. J Exp Med 210: 2087-2103. [Crossref]

21. Liu Y, Qin L, Wilson B, Wu X, Qian L, et al. (2008) Endotoxin induces a delayed loss of TH-IR neurons in substantia nigra and motor behavioral deficits. Neurotoxicology 29: 864-870. [Crossref]

22. Carlson CG, Rutter J, Bledsoe C, Singh R, Hoff H, et al. (2010) A simple protocol for assessing inter-trial and inter-examiner reliability for two noninvasive measures of limb muscle strength. J Neurosci Methods 186: 226-230. [Crossref]

23. Hosaka Y, Yokota T, Miyagoe-Suzuki Y, Yuasa K, Imamura M, et al. (2002) Alpha1syntrophin-deficient skeletal muscle exhibits hypertrophy and aberrant formation of neuromuscular junctions during regeneration. J Cell Biol 158: 1097-1107. [Crossref]

24. Ogawa N, Hirose Y, Ohara S, Ono T, Watanabe Y (1985) A simple quantitative bradykinesia test in MPTP-treated mice. Res Commun Chem Pathol Pharmacol 50: 435-441. [Crossref]

25. Olsson, M (1995) Forelimb akinesia in the rat Parkinson model: differential effects of dopamine agonists and nigral transplants as assessed by a new stepping test. $J$ Neurosci 15: 3863-3875.

26. Carter RJ, Lione LA, Humby T, Mangiarini L, Mahal A, et al. (1999) Characterization of progressive motor deficits in mice transgenic for the human Huntington's disease mutation. J Neurosci 19: 3248-3257. [Crossref]

27. Guyenet SJ, Furrer SA, Damian VM, Baughan TD, La Spada AR, et al. (2010) A simple composite phenotype scoring system for evaluating mouse models of cerebellar ataxia. J Vis Exp. [Crossref]

28. Halldorsdottir S, Carmody J, Boozer CN, Leduc CA, Leibel RL (2009) Reproducibility and accuracy of body composition assessments in mice by dual energy x-ray absorptiometry and time domain nuclear magnetic resonance. Int $J$ Body Compos Res 7: 147-154. [Crossref]

29. Horio F, Teradaira S, Imamura T, Anunciado RV, Kobayashi M, et al. (2005) The HND mouse, a nonobese model of type 2 diabetes mellitus with impaired insulin secretion. Eur J Endocrinol 153: 971-979. [Crossref]

30. Lee JY, Son HJ, Choi JH, Cho E, Kim J, et al. (2009) Cytosolic labile zinc accumulation in degenerating dopaminergic neurons of mouse brain after MPTP treatment. Brain Res 1286: 208-214. [Crossref]

31. Yin F, Banerjee R, Thomas B, Zhou P, Qian L, et al. (2010) Exaggerated inflammation, impaired host defense, and neuropathology in progranulin-deficient mice. $J$ Exp Med 207: 117-128. [Crossref]
32. McNeill TH, Koek LL (1990) Differential effects of advancing age on neurotransmitter cell loss in the substantia nigra and striatum of C57BL/6N mice. Brain Res 521: 107117. [Crossref]

33. Matsuura K, Kabuto H, Makino H, Ogawa N (1997) Pole test is a useful method for evaluating the mouse movement disorder caused by striatal dopamine depletion. $J$ Neurosci Methods 73: 45-48. [Crossref]

34. Tseng KY, Kargieman L, Gacio S, Riquelme LA, Murer MG (2005) Consequences of partial and severe dopaminergic lesion on basal ganglia oscillatory activity and akinesia. Eur J Neurosci 22: 2579-2586. [Crossref]

35. Tillerson JL, Miller GW (2003) Grid performance test to measure behavioral impairment in the MPTP-treated-mouse model of parkinsonism. $J$ Neurosci Methods 123: 189-200. [Crossref]

36. Brooks SP, Dunnett SB (2009) Tests to assess motor phenotype in mice: a user's guide. Nat Rev Neurosci 10: 519-529. [Crossref]

37. Blume SR, Cass DK, Tseng KY (2009) Stepping test in mice: a reliable approach in determining forelimb akinesia in MPTP-induced Parkinsonism. Exp Neurol 219: 208211. [Crossref]

38. Fujiwara H (1992) Comparative studies of sulpiride and classical neuroleptics on induction of catalepsy, locomotor activity, and brain dopamine metabolism in mice. Pharmacol Biochem Behav 41: 301-308. [Crossref]

39. Huang XF (2006) Dopamine transporter and D2 receptor binding densities in mice prone or resistant to chronic high fat diet-induced obesity. Behav Brain Res 175: 415419.

40. Cyr M, Beaulieu JM, Laakso A, Sotnikova TD, Yao WD, et al. (2003) Sustained elevation of extracellular dopamine causes motor dysfunction and selective degeneration of striatal GABAergic neurons. Proc Natl Acad Sci U S A 100: 1103511040. [Crossref]

41. Savastano DM, Covasa M (2005) Adaptation to a high-fat diet leads to hyperphagia and diminished sensitivity to cholecystokinin in rats. J Nutr 135: 1953-1959. [Crossref]

42. Woods SC, D'Alessio DA, Tso P, Rushing PA, Clegg DJ, et al. (2004) Consumption of a high-fat diet alters the homeostatic regulation of energy balance. Physiol Behav 83: 573-578. [Crossref]

43. Flatt JP (1995) Use and storage of carbohydrate and fat. Am J Clin Nutr 61: 952S-959S [Crossref]

44. Gallego M (2003) Diabetes-induced biochemical changes in central and peripheral catecholaminergic systems. Physiol Res 52: 735-741.

45. Papapetropoulos S, Ellul J, Argyriou AA, Talelli P, Chroni E, et al. (2004) The effect of vascular disease on late onset Parkinson's disease. Eur J Neurol 11: 231-235. [Crossref]

46. Morris JK, Zhang H, Gupte AA, Bomhoff GL, Stanford JA, et al. (2008) Measures of striatal insulin resistance in a 6-hydroxydopamine model of Parkinson's disease. Brain Res 1240: 185-195. [Crossref]

47. Figlewicz DP, Szot P, Chavez M, Woods SC, Veith RC (1994) Intraventricular insulin increases dopamine transporter mRNA in rat VTA/substantia nigra. Brain Res 644: 331-334. [Crossref]

48. Craft S, Watson GS (2004) Insulin and neurodegenerative disease: shared and specific mechanisms. Lancet Neurol 3: 169-178.

49. Roseberry AG, Painter T, Mark GP, Williams JT (2007) Decreased vesicular somatodendritic dopamine stores in leptin-deficient mice. J Neurosci 27: 7021-7027. [Crossref]

50. Figlewicz DP, Patterson TA, Johnson LB, Zavosh A, Israel PA, et al. (1998) Dopamine transporter mRNA is increased in the CNS of Zucker fatty (fa/fa) rats. Brain Res Bull 46: 199-202. [Crossref]

51. Lin S, Thomas TC, Storlien LH, Huang XF (2000) Development of high fat dietinduced obesity and leptin resistance in $\mathrm{C} 57 \mathrm{Bl} / 6 \mathrm{~J}$ mice. Int J Obes Relat Metab Disord 24: 639-646. [Crossref]

52. Ye SM, Johnson RW (1999) Increased interleukin-6 expression by microglia from brain of aged mice. J Neuroimmunol 93: 139-148. [Crossref]

53. Kohman RA, Bhattacharya TK, Wojcik E, Rhodes JS (2013) Exercise reduces activation of microglia isolated from hippocampus and brain of aged mice. $J$ Neuroinflammation 10: 114. [Crossref] 
Stojakovic A (2018) Long-term consumption of high-fat diet impairs motor coordination without affecting the general motor activity

54. Nakamura YK, Omaye ST (2012) Metabolic diseases and pro- and prebiotics: Mechanistic insights. Nutr Metab (Lond) 9: 60. [Crossref]
55. Puig KL, Floden AM, Adhikari R, Golovko MY, Combs CK (2012) Amyloid precursor protein and proinflammatory changes are regulated in brain and adipose tissue in a murine model of high fat diet-induced obesity. PLoS One 7: e30378. [Crossref]

Copyright: (C2018 Stojakovic A. This is an open-access article distributed under the terms of the Creative Commons Attribution License, which permits unrestricted use, distribution, and reproduction in any medium, provided the original author and source are credited. 